\title{
LARYNGOLOGY
}

\section{Office-based procedures in laryngology}

\section{Procedure ambulatoriali in laringologia}

\author{
Marta Filauro ${ }^{1,2,3}$, Alberto Vallin ${ }^{1,2}$, Marco Fragale ${ }^{1,2}$, Claudio Sampieri1,2, Luca Guastini ${ }^{1,2}$, Francesco Mora ${ }^{1,2}$, \\ Giorgio Peretti ${ }^{1,2}$ \\ ${ }^{1}$ IRCCS Ospedale Policlinico San Martino, Genoa, Italy; ${ }^{2}$ Department of Surgical Sciences and Integrated Diagnostics (DISC), \\ University of Genoa, Italy; ${ }^{3}$ Department of Experimental Medicine (DIMES), University of Genoa, Italy
}

\begin{abstract}
SUMMARY
Objective. Development of transnasal fiberoptic laryngoscopy, integration of an operative channel (OC), the advent of high-definition television imaging, with improvements in laser technology, cleared the way for office-based laryngology. Three treatment categories can be identified: bioendoscopy-guided biopsy; laryngeal injection; laser-assisted surgery.

Methods. 26 patients underwent OBPs at the Otolaryngology Clinic of IRCCS Policlinico San Martino, Genoa, Italy. Sixty-eight procedures were performed: 60 for recurrent respiratory papillomatosis (RRP), 5 for unilateral vocal fold paralysis (UVFP) and 3 for glottic leukoplakias. Neoblucaine 5\% was administrated through the operative channel, for local anaesthesia. All procedures were carried out with the physician standing behind the patient. Narrow band imaging (NBI - Olympus Medical) or i-scan (Pentax Medical) were used to enhance the accuracy of the biopsy thanks to identification of atypical vascular patterns. Laryngeal injections were made using a 25G flexible needle. Opera Evo (Quanta system IEC/EN 60825-1:2007) is a hybrid fibre laser that is used for "blanching" and vaporisation of RRP lesions and to treat selected leukoplakias that were previously biopsied.

Conclusions. No major complications occurred during the procedures.
\end{abstract}

KEY WORDS: laryngology, videolaryngoscopy, office-based procedures, injection, recurrent respiratory papillomatosis

\section{RIASSUNTO}

Oggetto. Lo sviluppo della fibrolaringoscopia transnasale, l'integrazione del canale operativo e l'avvento dell'alta definizione, associato ai miglioramenti della tecnologia laser, hanno permesso la diffusione delle procedure laringologiche eseguite ambulatorialmente. Esistono 3 categorie di trattamenti: biopsie guidate dalla bioendoscopia, iniezioni endolaringee e chirurgia laser-assistita.

Metodi. 26 pazienti sono stati sottoposti a trattamenti ambulatoriali presso la Clinica Otorinolaringoiatrica dell'IRCCS Policlinico San Martino di Genova, Italia. Sono state eseguite 68 procedure: 60 per papillomatosi respiratoria ricorrente, 5 per paralisi unilaterale della corda vocale e 3 per leucoplachie glottiche. Tutte le procedure sono state eseguite posizionandosi dietro il paziente. Per aumentare l'accuratezza della presa bioptica, abbiamo utilizzato la Narrow band imaging (NBI-Olympus Medical) o i-scan (Pentax Medical). Le iniezioni endolaringee sono state eseguite con un ago flessibile da 25G. Per eseguire la vaporizzazione delle lesioni papillomatose e per trattare le leleucoplachie selezionate ̀̀ stato utilizzato il laser a fibra ibrido Opera Evo (Quanta system IEC/EN 60825-1:2007). Conclusioni. Nessuna complicanza maggiore è incorsa durante le procedure.

PAROLE CHIAVE: laringologia, videolaringoscopia, ambulatorio, laringoplastica iniettiva, papillomatosis respiratoria ricorrente

\section{Introduction}

Office-based laryngology procedures (OBPs) emerged in the $19^{\text {th }}$ century; at that time, they were performed with the limits of indirect vision through laryngeal mirrors and by the paucity of instrumentation. When transnasal fibreoptic
Received: June 18, 2020

Accepted: July 28, 2020

Published online: December 29, 2020

\section{Correspondence \\ Marta Filauro}

Department of Surgical Sciences and Integrated Diagnostics (DISC), University of Genoa, Italy Department of Experimental Medicine (DIMES), University of Genoa, Italy

E-mail: mfilauro@yahoo.com

Funding

None.

Conflict of interest

The Authors declare no conflict of interest.

How to cite this article: Filauro M, Vallin A, Fragale $\mathrm{M}$, et al. Office-based procedures in laryngology. Acta Otorhinolaryngol Ital 2021;41:243-247. https://doi.org/10.14639/0392-100X-N0935

๑ Società Italiana di Otorinolaringoiatria e Chirurgia Cervico-Facciale

\section{(c) (1) $\$($}

This is an open access article distributed in accordance with the CC-BY-NC-ND (Creative Commons Attribution-NonCommercial-NoDerivatives 4.0 International) license. The article can be used by giving appropriate credit and mentioning the license, but only for non-commercial purposes and only in the original version. For further information: https:// creativecommons.org/licenses/by-nc-nd/4.0/deed.en 
laryngoscopy became available in 1975, it laid the groundwork for the shift from the operating room (OR) to the office ${ }^{1}$. Moreover, integration of an operative channel (OC) in the flexible endoscope widely extended the range of procedures that could be performed, especially those assisted by the appropriate type of laser beam delivered through fibre. Lastly, the advent of high-definition television fibreoptic imaging, together with improvements in laser technology, definitively cleared the way for the consolidation of office-based laryngology ${ }^{2}$.

Nowadays several treatments, broadly divided in three categories, can be performed in an office-based setting:

1. Bioendoscopy-guided biopsy with a more convenient approach compared to previous transoral access.

2. Laryngeal injection has benefited from the possibility of inserting an injecting needle through the OC as an alternative to a transcutaneous approach to administer laryngeal augmentation materials or drugs (i.e., hyaluronic acid, steroids, botulinum toxin, cidofovir, bevacizumab, etc.).

3. Laser-assisted surgery to address several selected benign or premalignant diseases (i.e., recurrent respiratory papillomatosis (RRP), leukoplakias). Different laser fibers are available on the market, each with specific characteristics of wavelength, delivery mode, safety and costs: proper selection of the type of laser is the key to avoid suboptimal results and undesirable side effects ${ }^{3}$.

This manuscript aims to give insight into our office-based laryngology setting and instrumentation and to define the type of pathologies that we currently treat with this approach.

\section{Methods}

In the time frame from May 2019 to February 2020, 26 patients underwent OBPs at the Otolaryngology Outpatient Clinic of IRCCS Policlinico San Martino, Genoa, Italy. A total of 68 procedures were performed.

An Olympus CV-170 ENT digital platform together with a videoendoscope ENF-VT2 (Olympus medical system) or Pentax Medical Defina EPK-3000 with Video Naso Pharyngo Laryngoscope VNL 1570STK (Pentax Medical) were alternatively used. Both videoendoscopes have an OC for the insertion of instruments.

Before each procedure, in order to obtain the best patient compliance, we applied a standardised protocol for administration of local anaesthesia. Before starting the procedure, $2 \mathrm{ml}$ of $5 \%$ neoblucaine is sprayed into the patient's nostril. (Fig. 1A and Fig. 2A). Subsequently, the physician gets into position behind the patient, inserting the videoendoscope into the nose (Fig. 2B). This setting is designed to keep the

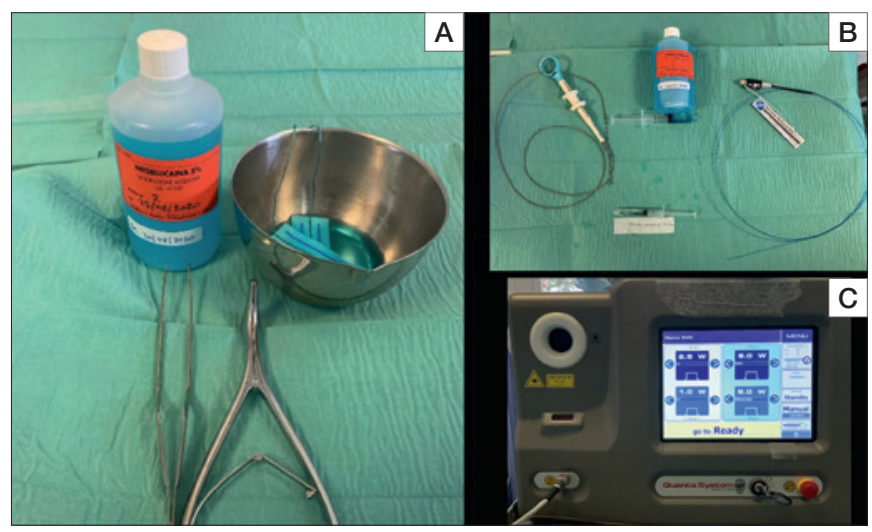

Figure 1. (A) Neoblucaine 5\%; (B) From the left side: endoscopic forceps, $5 \%$ neoblucaine, tullio-diode laser fibre and bevacizumab; (C) Opera Evo hybrid laser.

laryngologist in a safer position, avoiding direct exposure to nasal and oral secretions, while the patient can follow the procedure on the screen, enhancing compliance. Moreover, this modality of performing videolaryngoscopy, as routinely adopted by bronchoscopists, allows the physician to obtain the same laryngeal orientation and exposure achievable in the OR under general anaesthesia while maintaining full laryngeal visualisation. Finally, local anaesthesia is further administrated directly through the OC, just above laryngeal aditus, in order to spray an additional 3-4 $\mathrm{ml}$ of neoblucaine 5\% directly on the epiglottis, posterior commissure and glottis: to better convey the anaesthetic effects and prolong contact with the larynx, during the procedure patients are initially asked to sniff vigorously and to phonate immediately after (Fig. 2B). After a few minutes, the entire larynx is adequately anesthetised, and the surgical procedure can begin.

Biopsies were performed with specific flexible forceps through the OC (Fig. 2D). Narrow band imaging (NBI Olympus Medical) or i-scan (Pentax Medical) were used to select the proper site to enhance the accuracy of the biopsy thanks to the identification of atypical vascular patterns inside and surrounding suspicious lesions.

Laryngeal injections were made using a $25 \mathrm{G}$ flexible, dedicated needle (length of the tip of the needle $=5 \mathrm{~mm}$ ) through the OC (Fig. 1B and Fig. 3H). Hyaluronic acid was injected into the thickest part of the vocal ligament, between this and the thyroarytenoid muscle, for augmentation of vocal cord mass in case of unilateral vocal fold paralysis and bevacizumab (12.5 mg) was injected submucosally as adjuvant treatment for RRP. To avoid retaining of the material inside the injection needle and to guarantee that a full dose is administrated, $1 \mathrm{ml}$ of saline solution was introduced inside the needle at the end of each material injection. 

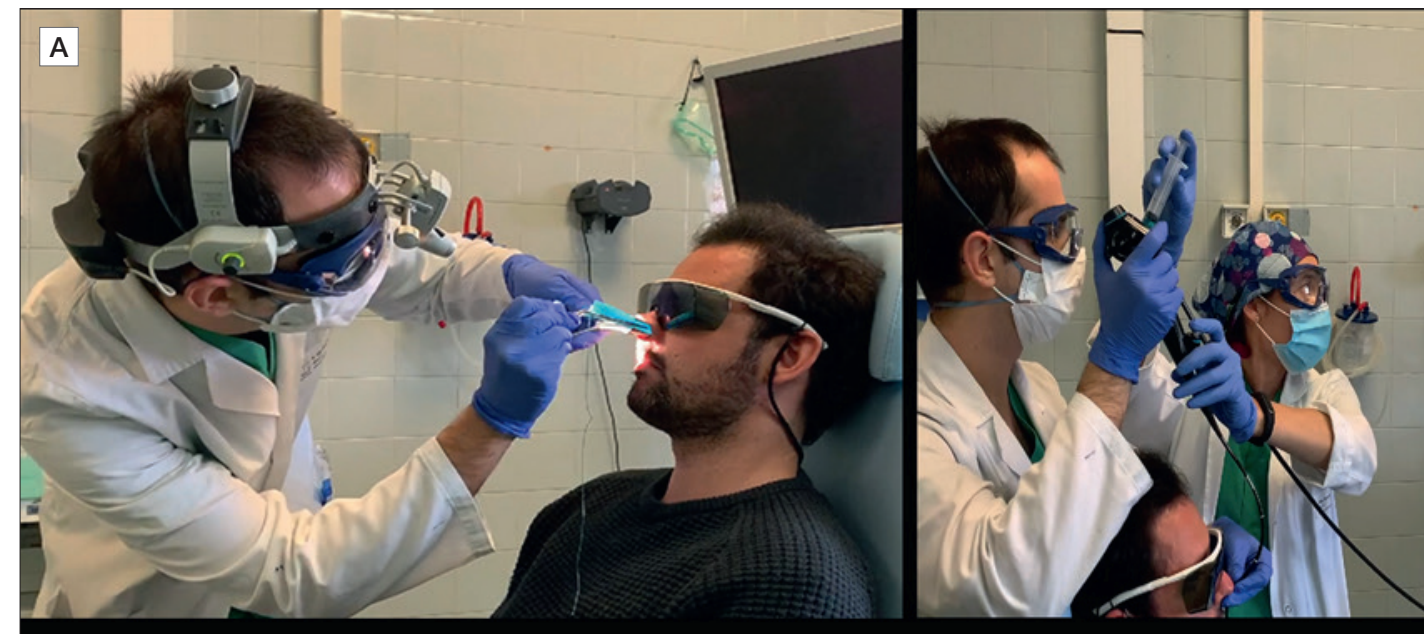

B
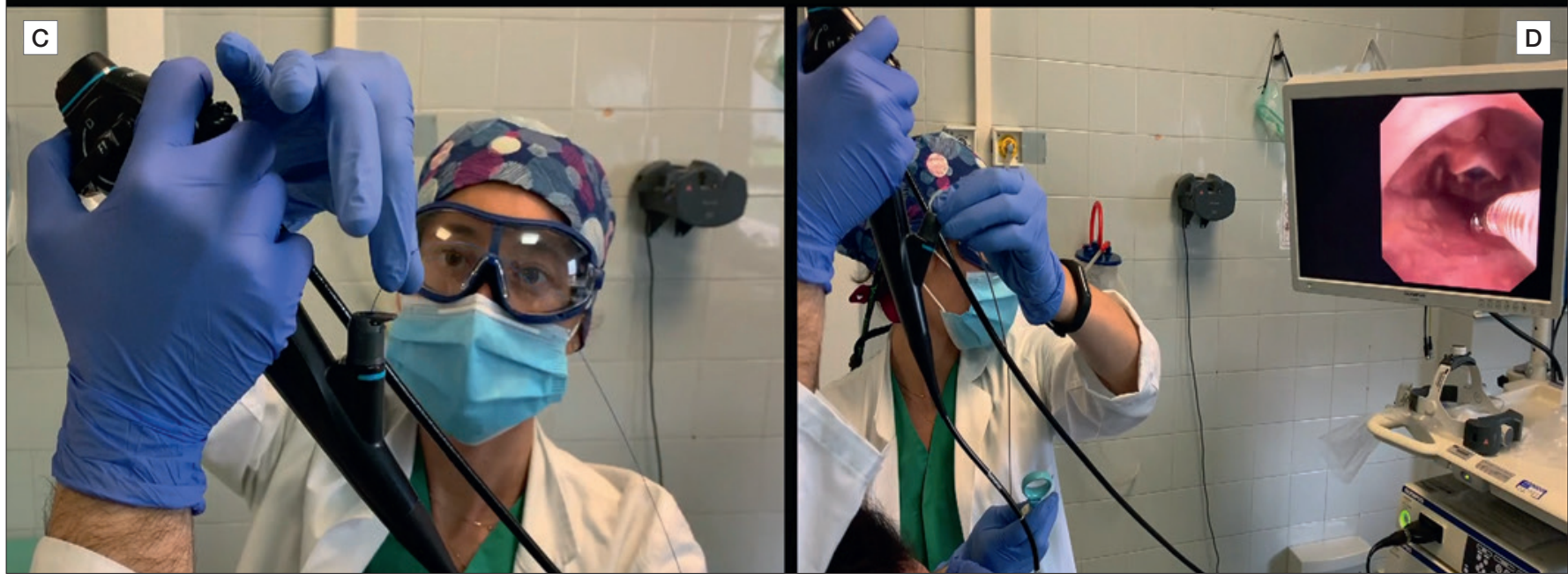

Figure 2. (A) Local anaesthesia into patient's nose; (B) Local anaesthesia through the operative channel; (C) Laser fibre insertionthrough the operative channel; (D) Endoscopic forceps insertion through the operative channel.

Opera Evo (Quanta system IEC/EN 60825-1:2007) is a hybrid fibre laser that is used for "blanching" and vaporisation of RRP lesions and to treat selected leukoplakias that were previously biopsied (Fig. 1B and Fig. 1C). This hybrid thulium/diode laser with a dualwavelength $(1.9 \mu \mathrm{m}$ and $1.5 \mu \mathrm{m})$ allowed for intraoperative fine-tuning of the instrument between cutting function, vaporisation and haemostasis. Pulsed emission is our preferred modality (ultrapulsed $4 \mathrm{~W}-1.5 \mathrm{~W}$ ), as it causes less thermal damage to the mucosa and the vocal ligament. Laser energy is transferred to the target by flexible fibres available in different calibers (200 $\mathrm{mm} ; 272 \mathrm{~mm} ; 365 \mathrm{~mm} ; 550 \mathrm{~mm} ; 800 \mathrm{~mm} ; 1000 \mathrm{~mm}$ ). In our experience, $200 \mathrm{~mm}$ and $272 \mathrm{~mm}$ diameters are the most suitable for OBPs as they fit perfectly in the OC. Each fibre is reusable for a maximum of 10 times. A full sterilisation process is carried out after each use.

\section{Results}

A total of 26 patients ( 17 males and 9 females) were treated. The mean age was 46.8 years (range 15-82). Of the 68 OBPs, 60 were performed for RRP, 5 for unilateral vocal fold paralysis (UVFP) and 3 for glottic leukoplakias. All 60 procedures carried out for RRP included laser vaporisation of papillomas and subsequent injection of bevacizumab submucosally (Figs. 3A, 3B, 3C). For RRP, the endoscopic treatment was repeated every 6 weeks, for a maximum of 6 times.

During the five injection laryngoplasties for UVFP, hyaluronic acid was injected inside the vocal ligament: this injection site, together with the high-density material, ensures that the hyaluronic acid remains in the vocal fold, allowing a long-lasting augmentation effect (Fig. 3H).

All three vaporisation procedures for leukoplakia were performed on patients who were unfit for transoral laser 


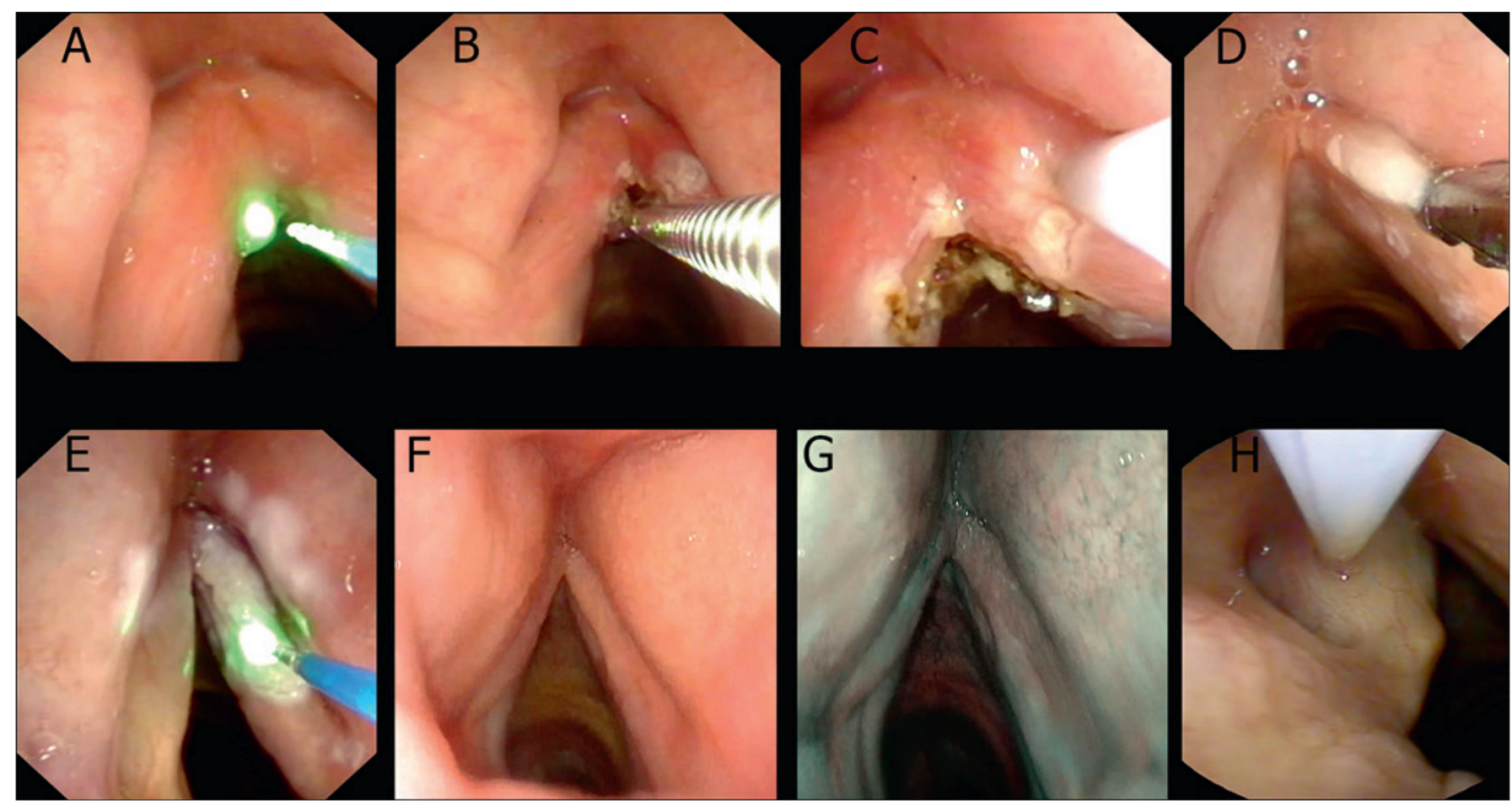

Figure 3. (A) Papilloma laser surgery; (B) Vapourised tissue asportation; (C) Submucosal bevacizumab injection; (D) Bioendoscopy-guided biopsy; (E) Glottic leukoplakia vaporisation; (F) Three-month white light follow-up; (G) Three-month NBl follow-up; (H) Hyaluronic acid injection into the vocal ligament.

microsurgery (TLM) under general anaesthesia due to inadequate exposure and/or poor general conditions. These endoscopic procedures were preceded by incisional biopsy of the lesion, which was also performed in an office-based setting (Fig. 3D) . During all the incisional biopsies, bioendoscopic tools, such as NBI ore iScan, were used in order to collect the sample in the most representative area. Only if the pathological report confirmed a diagnosis of mild or moderate dysplasia (LIN1-2) was office-based laser vaporisation carried out (Figs. 3E, 3F, 3G); otherwise, in case of diagnosis of squamous cell carcinoma, patients were submitted to radiotherapy, as TLM is unfeasible in terms of definitive and unimodal treatment. No major complications occurred during any procedure. None of the procedures had to be discontinued.

\section{Discussion}

Office-based procedures (OBPs) are a safe and reliable treatment modality for selected benign and premalignant laryngological diseases. Advances in anaesthesia, technology, instrumentation and surgical technique have resulted in a therapeutic shift from the operating room to the office, and it is estimated that up to $15 \%$ of procedures occur in an office-based setting ${ }^{4}$. The main reason for the widespread diffusion of OBPs is related to the possibility for patients to be discharged immediately after the procedure, avoiding hospitalisation. Secondly, waiting lists for procedures in general anaesthesia can be shortened without compromising outcomes and with significant cost reduction. Finally, the safety of frail patients is enhanced by preventing the risks associated with general anaesthesia. Above all, treatment of highly recurrent diseases, such as RRP, benefits from the advantages of OBPs such as the possibility to treat every small recurrence and/or persistence with a shorter interval of time between treatments, while maintaining good voice quality and adequate respiratory space and avoiding the growth of papillomas that would require an intervention under general anaesthesia. Furthermore, the office-based setting described herein seems to be particularly useful in RRP and in all cases of highly contagious airway diseases, allowing proper visualisation of all laryngeal subsites, including laryngeal ventricle and posterior commissure, and also places healthcare practitioners in a position of greater safety against airborne contamination by microorganisms. During OBPs, the awake patient provides real-time vocal outcomes, which is critical for fine-tuning of injection laryngoplasty. Furthermore, the disadvantages of direct laryngoscopy, such as dental damage or limitations in exposure, can be overcome thanks to the transnasal endoscopy ap- 
proach. On the other hand, ensuring that patients are able to tolerate the procedure is the key to ensuring success in this kind of surgery. Due to gag reflexes and movements, OBPs can be less precise than surgery performed under general anaesthesia, which may render the treatment less effective or cause iatrogenic injuries. To overcome this limitation, adequate local anaesthesia and continuous verbal contact with the patient can be useful. Cases with exophytic or bulky lesions, and airway compromise, are not ideal candidates for OBPs. Complications related to topical anaesthesia, like vasovagal or allergic reactions, are exceptional but still possible. In our series, we did not have any complications, but data from the literature ${ }^{5}$ point out that OBPs are not free from complications. The most commonly reported include oedema, purpura or vascular leakage, and laryngospasm. Oedema can occur if the laser energy is delivered to tissues surrounding the target lesion. Purpura and vascular leakage are well-known effects of photothermolysis, but in the vast majority of cases can be minimised by adjusting the time of exposure of each laser pulse. Laryngospasm can occur in hypersensitive patients: in these cases, swallowing and forced inspiration from the nose, with mouth closed, allow quick resolution of the laryngospasm without the need for further measures. In addition to patient-related complications, instrumentation-related issues can occur. Attention must be paid when inserting laser fibres inside the operative channel: an incorrect insertion angle of the glass laser fibre can lead to the damage of the working channel. On the other hand, endoscopic injecting needles natively carry tip protection, which is retractable at the time of injection, and avoids possible damage to the operative channel.

\section{Conclusions}

In our experience, OBPs are safe and reliable. Moreover, they increase patient quality of life. Better patient selection and standardised laser energy parameters based on lesion pathology and size may help decrease complications and failure rates.

\section{References}

1 Williams RG, Farquharson IM, Anthony J. Fibreoptic laryngoscopy in the assessment of laryngeal disorders. J Laryngol Otol 1975;89:299316. https://doi.org/10.1017/S0022215100080403

2 Zeitels SM, Burns JA, Franco RA, et al. Office-based treatment of glottal dysplasia and papillomatosis with the 585-nm pulsed dye laser and local anesthesia. Ann Otol Rhinol Laryngol 2004;113:265-276. https://doi.org/10.1177/000348940411300403

3 Wellenstein DJ, Schutte HW, Takes RP, et al. Office-based procedures for the diagnosis and treatment of laryngeal pathology. J Voice 2018;32:502-513. https://doi.org/10.1016/j.jvoice.2017.07.018

4 Pynnonen MA, Schmalbach CE. Office-based procedures in otolaryngology. Otolaryngol Clin North Am 2019;52:xv-xvi. https://doi. org/10.1016/j.otc.2019.03.001

5 del Signore AG, Shah RN, Gupta N, et al. Complications and failures of office-based endoscopic angiolytic laser surgery treatment. J Voice 2016;30:744-750. https://doi.org/10.1016/j.jvoice.2015.08.022 